\title{
IMPACTO DE LA INGESTA DE RESIDUOS PLÁSTICOS EN PECES*
}

\author{
Edward Werner Escobar Condor \\ Yuri Izquierdo Villasante \\ Andrea Macedo Riva \\ Gustavo Remuzgo Panduro \\ Investigadores independientes \\ Alberto Huiman Cruz \\ Peru Waste Innovation S.A.C
}

\begin{abstract}
Resumen: El presente estudio corresponde a una revisión bibliográfica sobre las investigaciones realizadas en base a la exposición de una diversidad de peces a diferentes concentraciones y tipos de residuos plásticos, ya sea a través de la ingesta directa, por exposición a ambientes contaminados o mediante su transferencia a través de la cadena trófica. En el desarrollo se presenta una descripción de los tipos y grados de afecciones que estos residuos ocasionan a los peces; asimismo, se ha incluido una comparación de los resultados obtenidos por algunos autores en sus experimentaciones. Producto del análisis realizado se ha llegado a la conclusión de que los residuos son fácilmente asimilables por los peces, independientemente de sus dimensiones, y que, por su diminuto tamaño, los microplásticos hacen las veces de vectores acuáticos capaces de transportar en su superficie materias orgánicas e inorgánicas potenciales de ocasionar daños a los organismos marinos que las absorban directa o indirectamente.
\end{abstract}

Palabras clave: contaminación marina, residuos plásticos, peces.

* Artículo elaborado en el marco del Curso de Especialización de Residuos del Ámbito Municipal y No Municipal, organizado por el INTE-PUCP y dictado en el 2018. 


\title{
Impact of the Ingestion of Plastic Waste in Fish
}

\begin{abstract}
The present research corresponds to a review on the investigations carried out based on the exposure of a diversity of fish to different concentrations and types of plastic waste, either through direct intake, exposure to contaminated environments or by transferring them through the trophic chain. In the development, a description of the types and degrees of affectations that this waste causes to the fish is presented; likewise, a comparison of the results obtained by some authors in their experiments has been included. As a result of the analysis carried out, it has been concluded that the waste is easily assimilated by fish, regardless of their size and that due to their small size, microplastics act as aquatic vectors capable of transporting organic and inorganic materials on their surface to cause potential damage to marine organisms that absorb them directly or indirectly.
\end{abstract}

Keywords: Marine Contamination, Plastic Waste, Fishes.

\section{Edward Werner Escobar Condor}

Ingeniero ambiental, magister en Gestión Pública, y Doctorando en Medio Ambiente y Desarrollo Sostenible por la Unidad Nacional Federico Villarreal. Con más de 10 años de experiencia en el sector público y privado en Gestión de Residuos Sólidos Municipales y no municipales, Economía Circular y valorización. Actualmente, Subgerente de Gestión Ambiental en la municipalidad de Pueblo Libre.

Correo electrónico: eco.werner@gmail.com

\section{Yuri Izquierdo Villasante}

Ingeniero de Industrias Alimentarias y con estudios de maestría en Ciencias Ambientales en la Universidad Nacional Agraria La Molina. Con estudios de Ingeniera Industrial en la Universidad Tecnológica del Perú. Especialista en fiscalización ambiental, procedimiento administrativo sancionador, producción más limpia, gestión y tratamiento de residuos sólidos. Expositor institucional en eventos organizados por ADEX, SNI, FONAM en temas de producción más limpia, fiscalización ambiental y gestión ambiental. Ha trabajado en el Ministerio de Transporte y Comunicaciones, en el Organismo de Evaluación y Fiscalizacion Ambiental (OEFA), SENATI, entre otros.

Correo electrónico: izquierdo194295@gmail.com 


\section{Andrea Macedo Riva}

Licenciada en Biología de la Universidad Ricardo Palma, con especialización en Gestión de Calidad, Medio Ambiente y Seguridad y Salud Ocupacional, Gestión y Mejora de Procesos y, Gestión Integral de Residuos Sólidos. Actualmente brinda servicios de consultoría en temas de Calidad y Ambiente.

Correo electrónico: andrea.macedoriva@gmail.com

\section{Gustavo Remuzgo Panduro}

Ingeniero Mecánico por la Universidad Nacional del Callao. Especialista en brindar soluciones de diseño, cálculo, fabricación y puesta en marcha de equipos para el transporte de carga aplicados a los sectores de minería, construcción, hidrocarburos, agroindustria y gestión ambiental; así como para equipos de gestión de residuos sólidos. Consultor independiente para el sector público y privado con amplia experiencia en la industria metalmecánica, carrocera y automotriz. Actualmente se desempeña como gerente general de GRP Ingeniería y Proyectos. -

Correo electrónico: gremuzgop@gmail.com

\section{Alberto Huiman Cruz}

Ingeniero Geógrafo, Magíster en Ciencias Ambientales con Mención en Gestión y Ordenamiento Ambiental del Territorio, y Doctorando en Ciencias Ambientales. Calificado como Experto Internacional de Residuos, por la International Solid Waste Association (ISWA). Expositor, representando al Perú, en tema de residuos sólidos en Costa Rica, El Salvador, Colombia y Argentina. Actual Gerente General de Peru Waste Innovation S.A.C.

Correo electrónico: alberto@pwi.com.pe 


\section{Introducción}

La aparición y acumulación generalizada de residuos plásticos en el ambiente ha llevado a una creciente preocupación mundial en la última década (Lönnstedt \& Eklöv 2016).

Se estima que la producción global de plástico es de alrededor de 300 millones de toneladas métricas por año, con una proyección de aumento de 20 millones de toneladas métricas por año (Plastics Europe 2013). En el ambiente marino la cantidad de plástico acumulado es aún incierto, sin embargo, se estima que en la actualidad hay más de 5 billones de piezas plásticas flotando en hábitats pelágicos (Eriksen et al. 2014).

A esto debe agregarse que los polímeros plásticos muestran una degradación biológica lenta, por lo que permanecen en el ambiente durante cientos a miles de años, donde se descomponen en pedazos más pequeños debido a la radiación ultravioleta, fuerzas físicas e hidrólisis; por lo tanto, las partículas de plástico se acumulan como pequeños fragmentos (microplástico $<5 \mathrm{~mm}$, mesoplástico 5 - $25 \mathrm{~mm}$, macroplástico > $25 \mathrm{~mm}$ ) (Romeo et al. 2015). Debido a su pequeño tamaño y persistencia en el ambiente, estos fragmentos pueden ser ingeridos por una variedad de organismos y varios estudios ya han informado sobre la ingestión de microplásticos para más de 100 especies de peces y mamíferos marinos (Lusher, McHugh \& Thompson 2013; Rochman et al. 2014; Fossi et al. 2014).

Adicionalmente, se debe mencionar los efectos negativos que generan para el ambiente marino y los organismos que habitan en él, esto incluye el enredo, la ingestión y la posible toxicidad (Eriksen et al. 2014).

Los riesgos de toxicidad provienen de componentes que son agregados al mismo material en el proceso productivo (bisfenol A, ftalatos, nonilfenol y ésteres de difenilo polibromados), de los químicos y contaminantes de su superficie (metales y contaminantes orgánicos persistentes) y de ciertos polímeros que funcionan como aditivos para conferirle estabilidad, durabilidad y resistencia a la degradación; sin embargo, estos no se unen al material y pueden filtrarse al ambiente una vez degradado el plástico. De este modo, ingresan a las células de los organismos e interactúan con las moléculas, causando interrupciones del sistema endocrino y a la fisiología del organismo (Alomar et al. 2017). 
En condiciones de laboratorio, algunos autores han informado que la captación de microplásticos puede ocurrir en especies bajo diferentes escenarios de exposición y han sugerido que es probable que ocurra una transferencia trófica de estos residuos (Rochman et al. 2013; Sá et al. 2018).

Además, varios estudios han demostrado que los organismos marinos pueden absorber microplásticos, a menudo con grandes consecuencias, ya que pueden acumularse en los tejidos, servir como vehículos para el transporte de patógenos, adsorber y acumular contaminantes tóxicos. Los microplásticos tienen el potencial de causar efectos adversos, como el cáncer, la actividad reproductiva dañada, la respuesta inmune disminuida y la malformación en animales y humanos (Auta, Emenike \& Fauziah 2017).

Son diversas las causas y consecuencias ocasionadas por la captación de estos residuos, motivo por el cual nace el interés de este trabajo, que busca determinar, en base a una revisión bibliográfica, los impactos que genera la ingesta de residuos plásticos en los peces.

\section{Metodología}

El presente estudio se desarrolló bajo la metodología de tipo descriptiva. Se realizaron revisiones de diversas investigaciones científicas enfocadas a estudiar la contaminación marina a través de los microplásticos (MPs) y sus impactos sobre la biota marina, específicamente sobre los peces, permitiendo contrastar conceptos y conclusiones de los diversos autores.

La revisión metódica de literaturas resulta ser un método replicable, científico y transparente que permite analizar y comprender la línea de investigación que se desea seguir para las discusiones y conclusiones, para, de esta manera, poder brindar aportes.

\section{Resultados y discusión}

Romeo et al. (2015) realizaron un estudio, que conformó la primera evidencia sobre la presencia de residuos plásticos en el contenido del estómago de tres especies de peces pelágicos comerciales (Xiphias gladius «pez espada», Thunnus thynnus «atún de aleta azul» y Thunnus alalunga «atún blanco»); sus resultados (Cuadro 1) determinaron la presencia de residuos plásticos en las tres especies analizadas, X. gladius $(12,5 \%)$, T. thynnus $(32,4 \%)$ y T. alalunga $(12,9 \%)$, que difirieron en tamaño para cada especie depredadora; mesoplástico (44,4\%), macroplástico y mesoplástico (31,3\%) y microplástico (75\%), respectivamente. Asimismo, analizaron que la presencia de dichos residuos podría ser explicado por la estrategia de alimentación oportunista para el caso de T. thynnus, ya que desarrolla la captura de presas en aguas poco 
profundas, donde los fragmentos de plásticos son más abundantes debido a su flotabilidad. Esta tendencia a la ingesta de residuos plásticos fue similar a la determinada en Mullus surmuletus «salmonete de roca» por Alomar et al. (2017), quienes estudiaron la ingesta, caracterización y respuesta enzimática a los microplásticos (Cuadro 1), en base a dos métodos de captura (arrastre de fondo y red de trasmallo); de sus resultados se obtuvieron que la longitud total e ingesta promedio por método de captura fue de 18,32 $\pm 0,22 \mathrm{~cm}, 0,32$ $\pm 0,04 \mathrm{MPs} /$ individuo $(23,50 \%)$ y 22,84 $\pm 0,28 \mathrm{~cm}, 0,68 \pm 0,10 \mathrm{MPs} /$ individuo $(37,4 \%)$, respectivamente; concluyendo que la velocidad de ingesta aumenta con la longitud del pez, tal y como se presenció en el estudio previo con Thunnus thynnus, $\mathrm{y}$, además, esto puede verse influido por la zona de captura; los fondos marinos son considerados como los sumideros de plásticos, por lo que se esperaría que exista una mayor ingesta en las especies demersales; no obstante, la ingesta resulta mayor o igual en las especies pelágicas que se alimentan en la columna de agua.

Del mismo modo, Baalkhuyur et al. (2018), al estudiar especies comerciales y no comerciales en el Mar Rojo, observaron una alta ingesta de microplásticos en los organismos que habitan los arrecifes de coral $(46,2 \%)$ y menor ingesta en los que habitan los pastos marinos (7,7\%), lo cual también fue justificado por la zona de alimentación; las especies mesopelágicas (pastos marinos) mostraron una baja ingesta debido a que estas solo migran a la superficie en la noche para alimentarse y luego regresan a su habitad natural. Finalmente, en un estudio realizado con tres especies comerciales, Dicentrarchus labrax «lubina», Diplodus vulgaris «mojarra» y Platichthys flesus «platija», en el estuario de Modego en Portugal, Bessa et al. (2018) también concordaron al determinar que la distribución vertical de los peces puede influir en el número de microplásticos ingeridos por el pez; encontrando niveles más altos de ingesta en la especie bentopelágica D. vulgaris (73\%), con un máximo de 14 microplásticos extraídos de un solo individuo, que en las especies demersales D. labrax (23\%) y P. flesus (13\%); similar tendencia fue registrada en otras especies comerciales de peces pelágicos del Canal de la Mancha, 36,5\% (Lusher et al. 2013), y en las especies pelágicas-neríticas de la Costa Mediterránea de Turquía (Güven et al. 2017). No obstante, en los drenajes y el estuario del Golfo de México se reportó un bajo porcentaje de ocurrencia en la ingesta de microplásticos entre los peces de agua dulce (8\%) y los peces marinos (10\%) (Phillips \& Bonner 2015), por lo que se podría decir que los rangos de ingestión pueden variar entre los estudios, los hábitats y las ubicaciones, relacionando la ingesta de microplásticos con las diferentes estrategias de alimentación de los peces.

Si bien la mayoría de estudios se enfoca en el tracto digestivo, por ser 
et al. 2018; Güven et al. 2017; Romeo et al. 2015), otras investigaciones en cangrejos (Uca rapax) y bivalvos (Mytilus edulis) han demostrado que los micro y nano plásticos pueden ser translocados fuera del tracto digestivo contaminando otros órganos (Brennecke et al. 2015; Browne et al. 2008). Debido a estas afirmaciones, Collard et al. (2017) realizaron un estudio en el mar mediterráneo, empleando Engraulis encrasicolus «anchoveta europea», y comprobaron la presencia de microplásticos en un $80 \%$ de los hígados examinados, concluyendo que esto podría deberse a un mecanismo de endocitosis o fagocitosis a través del lumen del tracto intestinal. Asimismo, en una investigación de Ding et al. (2018) realizada con Oreochromis niloticus «tilapia roja», expuestos a diferentes concentraciones de poliestireno, se observó la presencia de estos en varios tejidos del organismo como intestino, branquias, hígado y cerebro, reportándose una dependencia de la bioacumulación con respecto al tiempo de exposición.

Además de que los residuos pueden ser translocados, se debe agregar que la presencia de microplásticos genera una serie de respuestas enzimáticas, tal y como lo demostraron Antão et al. (2018) al investigar el estrés oxidativo y el daño lipídico oxidativo en las branquias e hígado de Dicentrarchus labrax «lubina» a diferentes concentraciones de mercurio $(\mathrm{Hg})$, microplásticos y la combinación de ambos; concluyendo que la presencia de microplásticos influye en la bioconcentración de $\mathrm{Hg}$ en las branquias y bioacumulación de este en el hígado, debido a que los microplásticos absorben el Hg del agua, actuando como una ruta adicional del metal y, a su vez, al liberar dicho metal producen un aumento de su acumulación en otros órganos como el hígado, causando estrés oxidativo en las branquias e hígado; además, a concentraciones más altas de microplásticos, se produce daño oxidativo lipídico por la inducción significativa de las enzimas catalasa (CAT), Glutatión S-transferasa (GST) y superóxido dismutasa (SOD). Estos resultados contrastan a los obtenidos previamente por Alomar et al. (2017), quienes no obtuvieron evidencia del estrés oxidativo y daño celular en el hígado de M. surmuletus; sin embargo, también visualizaron el aumento de la enzima GST, lo que sugiere la inducción del sistema de detoxificación del pez. Esta falta de evidencia podría sustentarse según Ašmonait et al. (2018), quienes emplearon a Oncorhynchus mykiss «trucha arcoiris» para estudiar los efectos de los microplásticos tras un periodo de ingesta de 4 semanas, sin embargo, los resultados obtenidos no pudieron detectar ningún efecto significativo sobre la barrera intestinal, por lo cual concluyeron que los efectos nocivos de los microplásticos en peces dependen de su especie y el periodo de exposición.

Además, Lu et al. (2016) analizaron la ingesta de microplásticos de distintos tamaños (5 $\mu \mathrm{m}$ y $20 \mu \mathrm{m}$ ) en Danio rerio "pez cebra» y los efectos 
REVISTA KAWSAYPACHA: SOCIEDAD Y MEdio AMBIENTE Nº 4 (2019)

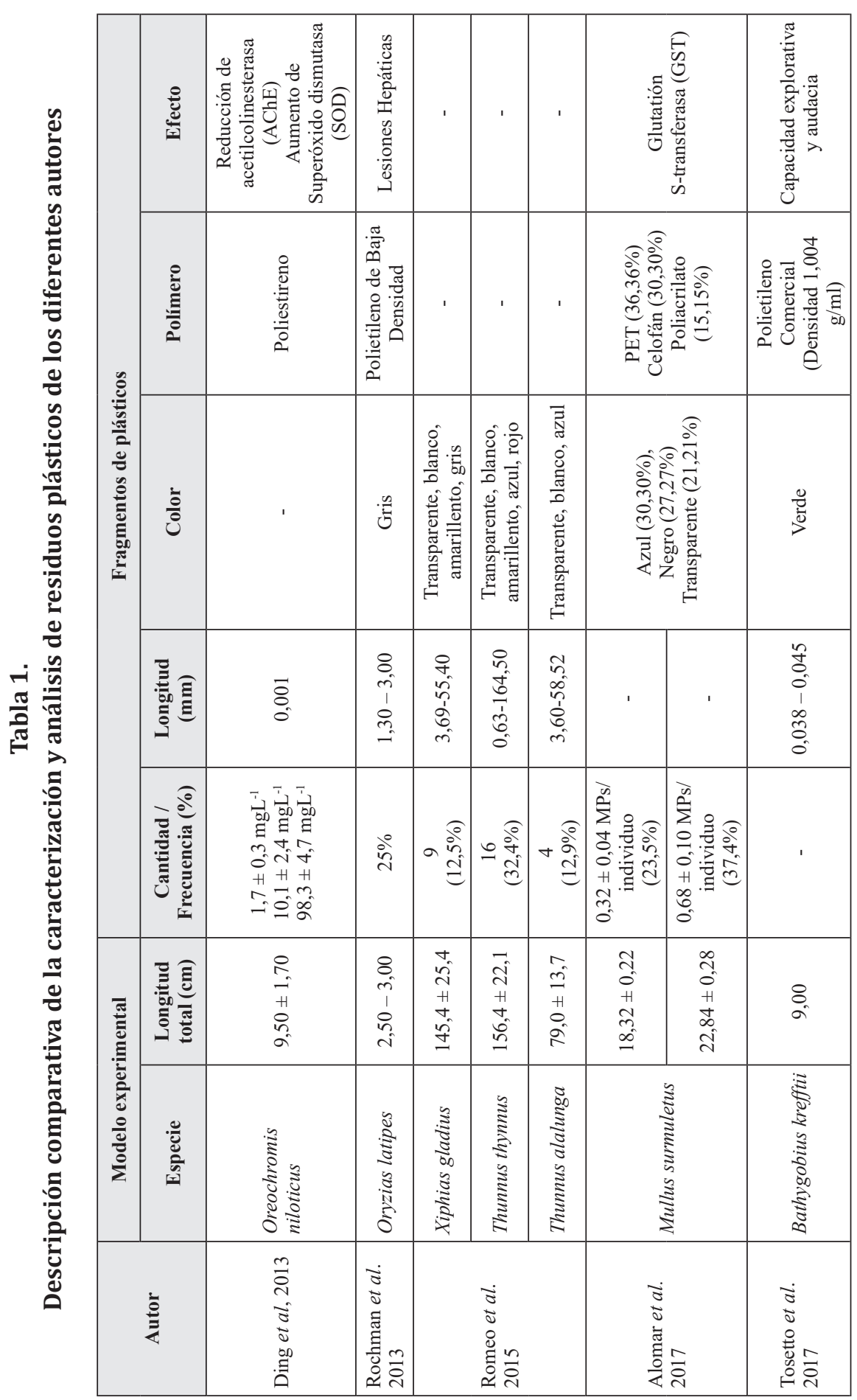


IMPACTO DE LA INGESTA DE RESIDUOS PLÁSTICOS EN PECES

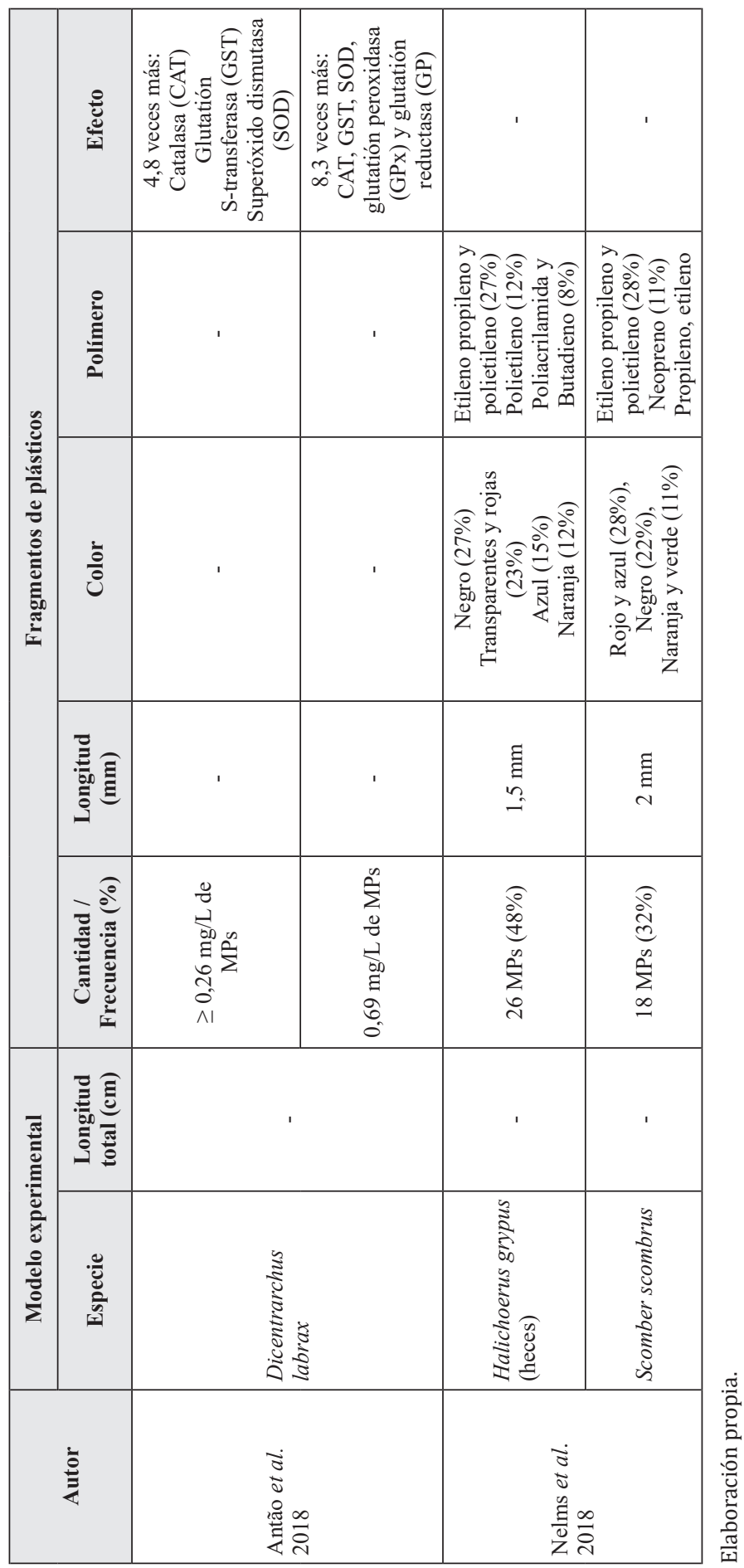


adversos a su exposición (mortalidad, reducción de la alimentación, inhibición del crecimiento, entre otros), observando la presencia de microplásticos en el tracto digestivo, independientemente del tamaño; sin embargo, esta variable se diferenció en el hígado y branquias, de menor y mayor tamaño, respectivamente.

Este resultado se complementa con lo descrito previamente por Romeo et al. (2015), quienes describieron que los microplásticos, según la forma y tamaño, pueden ubicarse en el interior del pez. Asimismo, Sá et al. (2018), al realizar una recopilación científica sobre los efectos nocivos de los microplásticos, llegaron a la conclusión de que los de menor tamaño llegan al hígado debido a la translocación de estos desde el intestino hasta el sistema circulatorio, lo cual explicaría lo descrito por Lu et al. (2016), que de igual forma señalaron que existe la posibilidad de que los sedimentos marinos puedan generar efectos sinérgicos con los microplásticos.

Asimismo, Tosetto, Williamson y Brown (2017), tras la aplicación de pruebas de personalidad a Bathygobius krefftii «gobios», alimentados con Platorchestia smithi «tolvas de playa» contaminadas con HAP (Hidrocarburos Aromáticos Policíclicos), determinaron que la personalidad no fue afectada en el corto plazo; sin embargo, durante los inicios de las pruebas de laboratorio, los peces mostraron relativa timidez, que fue asociada a la familiarización con los medios de prueba. Asimismo, el estudio encontró una mayor capacidad exploratoria de los peces luego de haberse sometido a las pruebas; el estudio tuvo una duración de 93 días, en vista de ello se recomendó efectuar análisis más prolongados; tal y como los efectuados por Rochman et al. (2013), donde el 25\% de los peces evaluados, Oryzias latipes «medaka japonesa», sufrieron insuficiencia hepática a través de lesiones superficiales, las cuales fueron evidenciadas recién 4 meses después de la exposición inicial; debido a que el polietileno (PBDE) en contacto con el medio marino contaminado resulta ser un vector potente para la bioacumulación de PBT (sustancias persistentes, tóxicas y bioacumulativas), como los HAP, PCB y PBDE.

Finalmente, Nelms et al. (2018) analizaron las heces de Halichoerus grypus «foca gris» y todo el tracto digestivo de Scomber scombrus «caballa» (Tabla 1), determinando que las partículas microplásticas pueden transferirse a través de niveles tróficos, sugiriendo una vía indirecta y potencialmente importante para la ingesta de microplásticos para cualquier especie cuya ecología de la alimentación implique el consumo de presas completas, pudiendo afectar a los humanos. 


\section{Conclusiones}

- Los resultados de la investigación señalan que los residuos son fácilmente asimilables por los peces, independientemente de sus dimensiones, y que, por su diminuto tamaño, los microplásticos hacen las veces de vectores acuáticos capaces de transportar en su superficie materias orgánicas e inorgánicas potenciales de ocasionar daños a los organismos marinos que las absorban directa o indirectamente.

- $\quad$ El tamaño de la ingesta de microplásticos está en función al tamaño del pez, donde a menor longitud, menor el tamaño del residuo; además, las especies pelágicas tienen mayor probabilidad de contaminarse con la ingesta de microplásticos por habitar en aguas medias o cerca de la superficie.

- $\quad$ Por otro lado, los fragmentos de plásticos más representativos en la ingesta de los peces según el color son: gris, verde, blanco, amarillento, azul, rojo, negro y transparente.

- Los resultados de investigaciones señalan hallazgos de microplásticos en el tracto digestivo, así como en heces de especies que forman parte de la cadena trófica.

- Las partículas de microplásticos pueden transferirse a través de niveles tróficos y su presencia en el cuerpo receptor genera una serie de respuestas enzimáticas que incluye la bioacumulación de mercurio (Hg), entre otros metales, y la combinación de ambos, lo que estaría causando estrés oxidativo. Por lo cual existe la probabilidad de transferir estos contaminantes por medio de niveles tróficos, lo que podría desencadenar en los consumidores finales enfermedades del tipo hepáticas, renales o nuevas enfermedades.

- Existe una alta probabilidad de exposición a los microplásticos transferidos por niveles tróficos, sobre todo para poblaciones que habitan cerca de las vías marítimas y próximas a áreas urbanas o industriales.

\section{Recomendaciones}

- En base a las metodologías estudiadas en la presente revisión y por la probabilidad de estar expuestos a una contaminación indirecta, se recomienda desarrollar investigaciones sobre la ocurrencia de la ingesta de residuos plásticos en especies comerciales a nivel de las zonas pesqueras identificadas a nivel nacional. 
- Reforzar las campañas de sensibilización poblacional sobre los riesgos y efectos de la contaminación marina a través de los residuos plásticos.

- $\quad$ Adoptar políticas de carácter vinculante en los gobiernos, los cuales permitan establecer controles para evitar el ingreso de plásticos a las playas del litoral peruano.

- De acuerdo a la relación entre el hábitat del pezy la cantidad de residuos plásticos ingeridos, se recomienda enfocar las investigaciones en especies comerciales pelágicas y demersales para identificar cuáles son las más y menos impactadas, y de esta forma adaptar nuestros hábitos de consumo como medida para minimizar el riesgo a una ingesta indirecta.

- $\quad$ En la mayor medida posible, tratar de consumir especies demersales como merluza, bagre, corvina, mero, lenguado, etcétera, ya que, según la bibliografía, son las menos impactadas.

\section{Referencias}

Alomar C., Sureda A., Capó X., Guijarro B., Tejada S., \& Deudero S. (2017). Microplastic ingestion by Mullus surmuletus Linnaeus, 1758 fish and its potential for causing oxidative stress. Environmental Research, 159: 135142. doi.org/10.1016/j.envres.2017.07.043

Antão L.G., Russo L., Branco V., Carvalho C., \& Guilhermino L. (2018). Microplastics increase mercury bioconcentration in gills and bioaccumulation in the liver, and cause oxidative stress and damage in Dicentrarchus labrax juveniles. Nature - Scientific Reports. 8 (1): 15655. doi:10.1038/s41598-018-34125-z pp.

Ašmonait G., Sundh H., Asker N., Bethanie M. \&, Almroth C. (2018). Rainbow trout maintain intestinal transport and barrier functions following exposure to polystyrene microplastics. Environ. Sci. Technol, 52 (24): 14392-14401. doi.org/10.1021/acs.est.8b04848

Auta, H., Emenike C., \& Fauziah S. (2017). Distribution and importance of microplastics in the marine environment: A review of the sources, fate, effects, and potential solutions. Environment International, 102: 165-176. doi.org/10.1016/j.envint.2017.02.013

Bahlkhuyur F., Dohaish E., Elhalwagy M., Alikunhi M., AlSuwailem A., Rostad A., Coker D., Berumen M., Duarte C. (2018). Microplastic in the gastrointestinal tract of fishes along the Saudi Arabian Red 
Sea coast. Marine Pollution Bulletin, 131: 407-415. doi.org/10.1016/j. marpolbul.2018.04.040

Bessa F.; Barría P.; Neto, João M.; Frias, João P.G.L; Otero, V.; Sobral, P.; MARQUES, J.C. (2018). Occurrence of microplastics in commercial fish from a natural estuarine environment. Marine Pollution Bulletin,128: 575584. /doi.org/10.1016/j.marpolbul.2018.01.044

Brennecke D., Ferreira E., Costa T., Appel D., Da Gama B. \&, Lenz M. (2015). Ingested microplastics $(>100 \mathrm{~mm})$ are translocated to organs of the tropical fiddler crab Uca rapax. Marine Pollution Bulletin, 96: 491-495.

Browne M., Dissanayake A., Galloway T., Lowe D., Thompson R. (2008). Ingested microscopic plastic translocates to the circulatory system of the mussel, Mytilus edulis (L.). Environ. Sci. Technol, 42(13): 4617-5032. doi. org/10.1021/es800249a

Collard F., Gilbert B., Compere Ph., Eppe G., Das K., Jauniaux Th., PARMENTIER E. (2017). Microplastics in livers of European anchovies (Engraulis encrasicolus, L.). Environmental Pollution, 229: 1000-1005. doi. org/10.1016/j.envpol.2017.07.089

Ding J., Zhang S., Mamitiana R., Zou H., Zhu W. (2018). Accumulation, tissue distribution, and biochemical effects of polystyrene microplastics in the freshwater fish red tilapia (Oreochromis niloticus). Environmental Pollution, 238: 1-9. doi.org/10.1016/j.envpol.2018.03.001

Eriksen, M., Lebreton, L.C.M., Carson, H.S., Thiel, M., Moore, C.J., Borerro, J.C., REISSER, J. (2014). Plastic pollution in the World's oceans: more than 5 trillion plastic pieces weighing over 250,000 tons afloat at sea. PLoS ONE 9(12): e111913. doi.org/10.1371/journal.pone.0111913

Fossi, M.C., Coppola, D., Baini, M., Giannetti, M., Guerranti, C., Marsili, L., Panti, C., De Sabata, E., Clò, S. (2014). Large filter feeding marine organisms as indicators of microplastic in the pelagic environment: the case studies of the Mediterranean basking shark (Cetorhinus maximus) and fin whale (Balaenoptera physalus). Marine Environmental Research, 100: 17-24. doi.org/10.1016/j.marenvres.2014.02.002

Güven, O., GöKdaĞ, K., Jovanović, B., Kideyş, A.E. (2017). Microplastic litter composition of the Turkish territorial waters of the Mediterranean Sea, and its occurrence in the gastrointestinal tract of fish. Environmental Pollution, 223: 286-294. doi.org/10.1016/j.envpol.2017.01.025

LÖNNSTEDT O. M. \& EKLÖV P. (2016). Environmentally relevant concentrations of microplastic particles influence larval fish ecology. Science, 352(6290): 1213-1216. doi: 10.1126/science.aad8828 
Lu Y., Zhang Y., Deng Y., Jiang W., Zhao Y., Geng J., Ding L., Ren L. (2016). Uptake and accumulation of polystyrene microplastics in zebrafish (Danio rerio) and toxic effects in liver. Environ. Sci. Technol., 50 (7): 4054-4060.

Lusher, A.L., McHugh, M., \& Thompson, R.C. (2013). Occurrence of microplastics in the gastrointestinal tract of pelagic and demersal fish from the English Channel. Mar. Pollut. Bull., 67(1-2): 94-99. doi.org/10.1016/j. marpolbul.2012.11.028

Nelms S.E., Galloway T.S., Godley B.J., Jarvis D.S., Lindeque P.K. (2018). Investigating microplastic trophic transfer in marine top predators. Environmental Pollution, 238: 999-1007. doi.org/10.1016/j. envpol.2018.02.016

Phillips, M.B. \& Bonner, T.H. (2015). Occurrence and amount of microplastic ingested by fishes in watersheds of the Gulf of Mexico. Marine Pollution Bulletin, 100(1): 264-269. doi.org/10.1016/j.marpolbul.2015.08.041

Plastics Europe (2013). Plastics - the Facts 2013. An analysis of European latest plastics production, demand and waste data. Belgium: Plastics Europe, Association of Plastic Manufacturers. Recuperado de: https://www. plasticseurope.org/application/files/7815/1689/9295/2013plastics_ the_facts_PubOct2013.pdf

Rochman, C.M., Hoh, E., Kurobe, T., Teh, S.J. (2013). Ingested plastic transfers hazardous chemicals to fish and induces hepatic stress. Scientific Reports, 3: 3263. doi:10.1038/srep03263

Rochman, C.M., Lewison, R.L., Eriksen, M., Allen, H., Cook, A.-M., Teh, S.J. (2014). Polybrominated diphenyl ethers (PBDEs) in fish tissue may be an indicator of plastic contamination in marine habitats. Science of the Total Environment. 476-477, 622-633. doi: 10.1016/j.scitotenv.2014.01.058

Romeo T., Pietro B., Peda C., Consoli P., Andaloro F., Fossi M.C. (2015). First evidence of presence of plastic debris in stomach of large pelagic fish in the Mediterranean Sea. Marine Pollution Bulletin, 95(1): 358-361. doi. org/10.1016/j.marpolbul.2015.04.048

Sá L. C., Oliveira M., Ribeiro F., Lopes T., Norman M. (2018). Studies of the effects of microplastics on aquatic organisms: What do we know and where should we focus our efforts in the future? Science of the Total Environment, 645: 1029-1039. doi.org/10.1016/j.scitotenv.2018.07.207

Tosetto, L.; Williamson, J.E., \& BRown, C. (2017). Trophic transfer of microplastics does not affect fish personality. Animal Behavior, 123: 159167. doi.org/10.1016/j.anbehav.2016.10.035 